\section{SuRface Salinity, Visualization, CD-ROMS}

$\mathrm{T}$ HE FACT THAT WE MAY soon be able to virtually walk (or run) through our data is exciting. I have often felt that support for oceanography would be better if people could see ocean currents, river plumes, and herds of whales as easily as they can see birds, clouds, and cold fronts. Imagine sitting on the bottom of a virtual ocean looking up through variably translucent density layers ... then you touch the control panel tweaking the trade winds a bit and watch the resulting adjustment. Imagine seeing the ocean from the viewpoint of a fish larvae or an oyster. The possibilities for research and education are nearly limitless. Wheless, Valle-Levinson, and Sherman describe some of the interesting aspects of virtual reality when applied to oceanography. Hopefully we all may soon be able to walk into a CAVE and explore a virtual ocean.

When Gary Lagerloef brought up the idea of a paper on remote sensing of salinity I was skeptical while at the same time knew that we desperately needed such measurements. His paper with Swift and Le Vine shows that the capability to measure interannual variability of surface salinity is near. Such measurements will no doubt help us understand the interplay between North Atlantic salinity and Deep Water formation. A sidebar piece by Jerry Miller describes an airplane mounted sensor that is nearly operational for observation of salinity in coastal waters.

This issue ends with Korgen's comments on the 1993 Briscoe and Evans article, Elizabeth Smith's review of CD-ROM technology in oceanography, and two Futures pieces one on coccolithophores by Christopher Brown and a second by Thomas Orsi on seafloor sediments.

- Larry Atkinson

\section{WE'VE MOVED. . .}

TOS Headquarters has moved to a new office. You can now reach us at:

\author{
4052 Timber Ridge Drive \\ Virginia Beach, VA 23455 \\ USA
}

Phone: (804) 464-0131; FAX: (804) 464-1759; Internet: jrhodes@ccpo.odu.edu

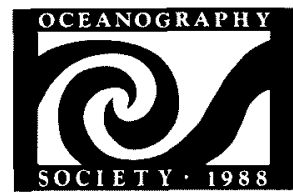

THE OCEANOGRAPHY SOCIETY

4052 Timber Ridge Drive Virginia Beach, VA 23455 USA (804) 464-0131; fax: (804) 464-1759

OFFICERS

Margaret Leinen, President Robert A. Duce, President-Elect Melbourne G. Briscoe, Secretary David Evans, Treasurer Arnold L. Gordon, Past-President

COUNSELORS

Richard T. Barber

Stephen E. Calvert

Tommy D. Dickey

Robert F. Anderson

Rick Spinrad

EXECUTIVE DIRECTOR Judi Rhodes

CORPORATE/NSTITUTIONAL SPONSORS Aanderaa Instruments, Inc., Woburn, MA, USA Chishitsu Chosa-Jo, ibaraki, Japan GE Astro Space Division Princeton, NJ, USA John G. Shedd Aquarium, Chicago, IL, USA

Monterey Bay Aquarium Research Institute, Pacific Grove, CA, USA

National Marine Fisheries Service, Silver Spring, MD, USA

Ober, Kaler, Grimes \& Shriver Washington, DC, USA

RD Instruments, San Diego, CA, USA Scripps Institution of Oceanography, La Jolla, CA, USA

Woods Hole Oceanographic Institution, Woods Hole, MA, USA

\section{OCEANOGRAPHY}

CO-EDITORS

Larry P. Atkinson

Center for Coastal Physical Oceanography

Old Dominion University

Crittenton Hall

Norfolk, VA 23529

(804) $683-5558$

Internet: atkinson@ccpo.odu.edu

Connie Sancetta

National Science Foundation

Ocean Sciences Division Room 725

4201 Wilson Blvd.

Arlington, VA 22230

(703) 306-1586

Internet: csancett@nsf.gov

$$
\begin{gathered}
\text { ASSOCIATE EDITORS } \\
\text { James W. Ammerman } \\
\text { Department of Oceanography } \\
\text { Texas A\&M University } \\
\text { College Station, TX 77843 USA } \\
\text { (409) 845-5105 } \\
\text { Gregg J. Brunskill } \\
\text { Australian Institute of Marine Science } \\
\text { PMB No. 3, Townsville, M.C. } \\
\text { Queensland 4810, Australia } \\
\text { (077) 789 211; FAX (077) 725 852; } \\
\text { Internet: g_brunskill@aims.gov.au }
\end{gathered}
$$

